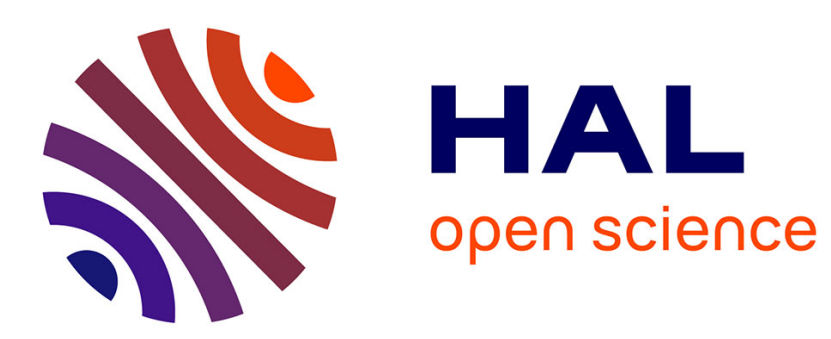

\title{
Sedimentation instabilities: Impact of the fluid compressibility and viscosity
}

Michael Niebling, Eirik Grude Flekkøy, Knut Jørgen Måløy, Renaud Toussaint

\section{To cite this version:}

Michael Niebling, Eirik Grude Flekkøy, Knut Jørgen Måløy, Renaud Toussaint. Sedimentation instabilities: Impact of the fluid compressibility and viscosity. Physical Review E: Statistical, Nonlinear, and Soft Matter Physics, 2010, 82 (5), 10.1103/PhysRevE.82.051302 . insu-02377739

\section{HAL Id: insu-02377739 \\ https://hal-insu.archives-ouvertes.fr/insu-02377739}

Submitted on 24 Nov 2019

HAL is a multi-disciplinary open access archive for the deposit and dissemination of scientific research documents, whether they are published or not. The documents may come from teaching and research institutions in France or abroad, or from public or private research centers.
L'archive ouverte pluridisciplinaire HAL, est destinée au dépôt et à la diffusion de documents scientifiques de niveau recherche, publiés ou non, émanant des établissements d'enseignement et de recherche français ou étrangers, des laboratoires publics ou privés. 


\title{
Sedimentation instabilities: Impact of the fluid compressibility and viscosity
}

\author{
Michael J. Niebling, ${ }^{1}$ Eirik G. Flekkøy, ${ }^{1}$ Knut Jørgen Måløy, ${ }^{1}$ and Renaud Toussaint ${ }^{2}$ \\ ${ }^{1}$ Department of Physics, University of Oslo, P.O. Box 1048, 0316 Oslo, Norway \\ ${ }^{2}$ Institut de Physique du Globe de Strasbourg, CNRS, and University of Strasbourg(EOST), 5 rue Descartes, \\ 67084 Strasbourg Cedex, France \\ (Received 18 May 2010; published 11 November 2010)
}

\begin{abstract}
The effect of an interstitial fluid on the mixing of sedimenting grains is studied numerically in a closed rectangular Hele-Shaw cell. We investigate the impact of the fluid compressibility and fluid viscosity on the dynamics and structures of the granular Rayleigh-Taylor instability. First we discuss the effect of the fluid compressibility on the initial fluid pressure evolution and on the dynamics of the particles. Here, the emerging patterns do not seem highly affected by the compressibility change studied. To characterize the patterns and motion the combined length of the particle trajectories in relation to the movement of the center of mass is analyzed, and the separation of particle pairs is measured as a function of the fluid viscosity.
\end{abstract}

DOI: 10.1103/PhysRevE.82.051302

PACS number(s): 45.70. $-\mathrm{n}, 45.50 .-\mathrm{j}, 47.11 .-\mathrm{j}$

\section{INTRODUCTION}

Granular materials play an important role in geological processes, for example, in erosion or avalanche processes [1-3]. Handling granular materials is in addition the daily basis of many of our industries, such as mining, agriculture, civil engineering, and pharmaceutical manufacturing. The field of dry granular materials and the enormous richness and complexity of granular motion and granular flows have provided the research of the last 20 years with numerous questions. In granular flows the presence of an interstitial fluid has been shown to strongly affect the dynamics of the grains [4-8]. The fluid compressibility and the fluid viscosity may well have an effect on the dynamics of the grains, for example, in fluidized bed reactors, but have not been in much focus.

The Rayleigh-Taylor instability in granular fluid mixtures has been studied with different types of fluids. With air the influence of the grain size was systematically investigated [9-11], and the behavior of this instability was compared between the cases where the interstitial fluid was air or water-glycerol [12]. In a comparable study of the SaffmanTaylor instabilities for granular-fluid mixtures oil or air was utilized as the interstitial fluid $[13,14]$. In our previous work [12] we have found in experiments and simulations that the mixing of the grains with the fluid during the granular Rayleigh-Taylor instability was very different whether we used air or a water-glycerol solution as the interstitial fluid. In pursuit to study this influence we further developed a numerical model $[9,10,13,15]$ that was proven $[12]$ to reproduce well the experimentally measured dynamics of the grains in the presence of a fluid. Even though we could give a quantitative explanation with the characterization of the correlation lengths and velocity field histograms, it is still an open question if the mixing behavior is a result of the fluid grain coupling to a compressible or incompressible fluid and how big is the influence of the fluid viscosity. To further answer this question we will perform numerical simulations with the goal to study the effect of the fluid viscosity and fluid compressibility on the mixing behavior during sedimentation. For this purpose the fluid viscosity and fluid com- pressibility are systematically and independently varied. These simulations show that the fluid compressibility has a small effect on the mixing except in extreme cases. The change in the fluid viscosity leads to an increase in the averaged particle trajectory length as a function of the displacement of the center of mass of the particles. With respect to the separation of particle pairs, the change in the viscosity displays initially two regimes: a nonhydrodynamic or ballistic regime at low fluid viscosities and a hydrodynamic regime at high fluid viscosities, with a crossover from diffusive to turbulent-dispersive behavior. This distinction however does not hold for the later stages of the simulations.

The paper is organized as follows. In the next section the implementation of the numerical model is briefly discussed. For more details and explanations see $[9,10,12,13,15]$. The results of the simulations with varied fluid compressibility and viscosity are presented in Sec. III. In Secs. III A and III B the effect of the fluid compressibility is studied. The effect of the fluid viscosity is studied and discussed in Sec. III C followed by the conclusions in Sec. IV.

\section{THEORY AND SIMULATIONS}

The numerical model is a two-dimensional hybrid model that uses a continuum description for the fluid and a discrete description of the granular phase. Friction between particles or the particles and the side plates is neglected. Further we neglect the friction between the fluid and the side plates. The model is derived in detail in $[9,10,12,13,15]$. It was tested and shown to reproduce the dynamics of granular flows at low Reynolds numbers, and we will only present the main equations for the evolution of the nonhydrostatic part of the pressure field $P$ and the dynamics of the particles briefly.

\section{A. Dynamics of the fluid}

The equations ruling the evolution of the nonhydrostatic part of the pressure, also termed the hydraulic head $P$, are derived in detail in Ref. [15]. The nonhydrostatic part of the pressure, $P$, corresponds to $P=P^{\prime}-\rho_{f} g y^{\prime}$, where $P^{\prime}$ is the pressure, $g$ is the gravity constant, $\rho_{f}$ is the fluid density, and 
$y^{\prime}$ is the depth. In the following we will only present and discuss the main equations briefly. We start with mass conservation of the fluid:

$$
\partial_{t}\left[\rho_{f}(P) \phi\right]+\nabla \cdot\left[\rho_{f}(P) \phi \mathbf{v}_{\mathbf{f}}\right]=0,
$$

where $\mathbf{v}_{\mathbf{f}}$ is the velocity of the fluid and $\phi$ is the local porosity: for the mass conservation of the grains we get

$$
\partial_{t}(1-\phi)+\nabla \cdot[(1-\phi) \mathbf{u}]=0,
$$

where $\mathbf{u}$ is the velocity of the grains. The velocity $\mathbf{v}_{\mathbf{f}}$ of the fluid is the sum of the local velocity relative to the grains, derived from Darcy's law, plus the velocity of the grains:

$$
\mathbf{v}_{\mathbf{f}}=\mathbf{u}-\frac{\kappa}{\phi \mu} \nabla P
$$

where $\mu$ is the fluid viscosity and $\kappa$ is the local permeability. Using Eq. (3) in Eq. (1) we get:

$$
\partial_{t}\left[\phi \rho_{f}(P)\right]+\nabla \cdot\left[\phi \rho_{f}(P)\left(\mathbf{u}-\frac{\kappa}{\phi \mu} \nabla P\right)\right]=0 .
$$

Eliminating $\partial_{t} \phi$ between Eqs. (2) and (4) and taking the fluid mass density $\rho_{f}(P)$ to be related to the pressure by the compressibility $\beta_{T}=-(1 / V) \partial V / \partial P$ through an equation of state that we linearize around the background pressure $P_{0}$ with $\rho_{f}^{0}$ as the fluid mass density at $P_{0}$,

$$
\rho_{f}(P) \approx \beta_{T} \rho_{f}^{0}\left(P-P_{0}\right)+\rho_{f}^{0},
$$

we get after a short calculation a diffusion equation for the nonhydrostatic part of the pressure $P$. This equation in general implies the fluid compressibility $[15,16]$ :

$$
\phi\left[\frac{\partial P}{\partial t}+\mathbf{u} \cdot \nabla P\right]=\nabla \cdot\left[\hat{P}\left(\beta_{T}\right) \frac{\kappa}{\mu} \nabla P\right]-\hat{P}\left(\beta_{T}\right) \nabla \cdot \mathbf{u},
$$

where we have defined $\hat{P}\left(\beta_{T}\right)=\rho_{f} /\left(\rho_{f}^{0} \beta_{T}\right)=P-P_{0}+1 / \beta_{T}$.

Since in this work we only simulate fluids with the mass density of air as the interstitial fluid, we can neglect the inertia and the weight of the fluid, and the hydraulic head correspond to the local pressure: $P^{\prime} \approx P$. In the case of air, considered as an ideal gas we get $\beta_{T}=1 / P_{0}$ at $P_{0}$ and Eq. (6) results in

$$
\phi\left[\frac{\partial P}{\partial t}+\mathbf{u} \cdot \nabla P\right]=\nabla \cdot\left(P \frac{\kappa}{\mu} \nabla P\right)-P \nabla \cdot \mathbf{u} .
$$

At the other end of fluid compressibility types, the incompressible limit where $\beta_{T} \rightarrow 0$, Eq. (6) results in a Poisson equation:

$$
\nabla \cdot\left(\frac{\kappa}{\mu} \nabla P\right)=\nabla \cdot \mathbf{u}
$$

In both cases we calculate the local permeability $\kappa$ by the Carman-Kozeny relation [17]

$$
\kappa=\frac{a^{2}}{9 K} \frac{\left(1-\rho_{s}\right)^{3}}{\rho_{s}^{2}},
$$

where $\rho_{s}=1-\phi$ is the solid volume fraction, $a$ is the particle radius, and $K=5$ is an empirical constant valid for a packing of beads [15].

\section{B. Dynamics of the particles}

The force equation for a single particle with the velocity $v_{p}$, particle mass $m=\rho_{m} \pi a^{2} h$, particle mass density $\rho_{m}$, volume $V_{a}=\pi a^{2} h$ in a cell with a plate spacing of $h$, and the number density defined as $\rho_{n}=\rho_{s} \rho_{m} / m$ is given by

$$
m \frac{d v_{p}}{d t}=\rho_{\mathrm{eff}} V_{a} \mathbf{g}+\mathbf{F}_{\mathbf{I}}-\frac{\nabla P}{\rho_{n}}+\mathbf{F}_{\mathbf{d}},
$$

where $\rho_{\text {eff }}=\rho_{m}-\rho_{f}$ enters in the buoyancy forces in the first term of the left-hand side, $\mathbf{F}_{\mathbf{I}}$ is the interparticle solid contact force, the third term arises from the momentum exchange between the fluid and solid, and $\mathbf{F}_{\mathbf{d}}$ is a viscous force accounting for energy dissipation. To approximate a situation of hard spheres we choose the interparticle force $\mathbf{F}_{\mathbf{I}}$ be a linear force with a spring constant $k$ high enough to make the overlap of particles during collision a negligible fraction of their diameters.

If particles collide we include energy dissipation with a restitution coefficient of $r_{s}=0.13$ [10]. This is modeled by a viscous force, active only during particle contact. This force is proportional to the relative velocity of the particles $\mathbf{v}_{\mathbf{r}}$ and oriented along the unit vector $\mathbf{n}_{\mathbf{d}}$, which points from the center of one particle to the contact point:

$$
\mathbf{F}_{\mathbf{d}}=-\gamma_{d}\left(\mathbf{v}_{\mathbf{r}} \cdot \mathbf{n}_{\mathbf{d}}\right) \mathbf{n}_{\mathbf{d}} .
$$

The particle propagation is modeled by the velocity Verlet scheme $[18,19]$.

\section{RESULTS}

For the simulations presented in this paper we used a Hele-Shaw cell in the $x$ direction of $\Delta x=5 \mathrm{~cm}$ in width and in the $y$ direction $\Delta y=7 \mathrm{~cm}$ in height. The cell is entirely closed on all sides, both for the fluid and for the grains. The mass density of the fluid was constant, set to the mass density of air $\rho_{f}=1.29 \mathrm{~kg} / \mathrm{m}^{3}$, and we considered particles of a mass density of $\rho_{m}=2500 \mathrm{~kg} / \mathrm{m}^{3}$. The initial pressure considered corresponds to atmospheric pressure, $P_{0}=100 \mathrm{kPa}$ at the top of the cell. The average size of the particles is $140 \mu \mathrm{m}$ and to avoid the formation of a triangular lattice the particle diameter was defined to have a flat size distribution with a range of $\pm 10 \%$ variation about the mean. In total approximately 140000 particles are considered in the simulations. The only variables that we vary in the following are the viscosity of the fluid $\mu_{f}$ and the compressibility of the fluid $\beta_{T}$. The initial state is prepared in the following way: particles are first let to rest on the bottom up to when a fraction of $2 / 3$ of the cell volume is filled. The interparticle space is filled with the interstitial fluid considered. Then, gravity is instantaneously reverted, corresponding to a sudden upside down flipping of the cell, and particles start to fall 


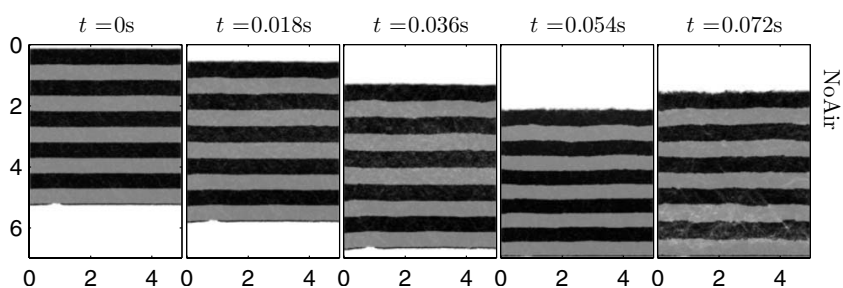

FIG. 1. A layer of beads falls through a gap in vacuum. Time progresses from left to right. White areas represent areas where the particle density is zero. The gray and black areas represent areas filled with particles, and the stripes are added artificially to better demonstrate the dynamics. From left to right time progresses in equal time steps.

from their initial configuration and initial zero velocity. In all the simulations the cell could be divided into three zones independent of the type of fluid. In the base of the cell, where particles have sedimented, and in the top section we find a bulk of compacted particles. In these two sections particles are hardly moving relative to each other. In between these two sections, we find a dynamic section with mobile dispersed particles. The particles in this section move while forming fingers of higher particle density and bubbles of lower particle density.

\section{A. Effect of the compressibility on the granular Rayleigh- Taylor instability}

The dynamics of the particles in the Hele-Shaw cell significantly depends on the interstitial fluid. In vacuum we do not see any evolution of patterns in the density field as shown in Fig. 1. Since all particles start at zero velocity, indeed, they simply all homogeneously fall in free fall, up to the moment when they contact the lower boundary and bounce back. In Fig. 2 an interstitial fluid however is present. While falling through the gap of fluid, here the particles develop downward falling fingers of high particle density and rising bubbles of low particle density. In this section we are going to investigate the effect of the fluid compressibility on the dynamics of the particles. In Fig. 2 we therefore vary and compare the dynamics for different fluid compressibilities. While the fluid viscosity is kept fixed and set to $\mu_{f}$ (air) $=0.018 \mathrm{mPa} \mathrm{s}$, which corresponds to air at room conditions of $25{ }^{\circ} \mathrm{C}$ and atmospheric pressure, the bulk modulus varied from $\kappa_{T}=1 / \beta_{T}=1 \mathrm{kPa}$ to incompressible behavior.

From the plots of the density field in Fig. 2 we can identify two main differences due to the change in compressibility. First we find for the high compressible cases with a bulk modulus of $\kappa_{T} \leq 5 \mathrm{kPa}$ that at times $t \leq 0.12 \mathrm{~s}$ bubbles of low particle density appear at the upper end of top section and right above the fingers in Fig. 2 in the picture at $t$ $=0.126 \mathrm{~s}$ in the top row. For a higher bulk modulus these bubbles are not present, and the top section stays uniformly compacted. Second we notice that in the beginning the center of mass of the whole top section moves further down the more compressible is the fluid. This movement of the top section stops when the weight of the packing is balanced by the overpressure of the compressed air in the base and the underpressure in the upper part of the cell. In Fig. 3 we

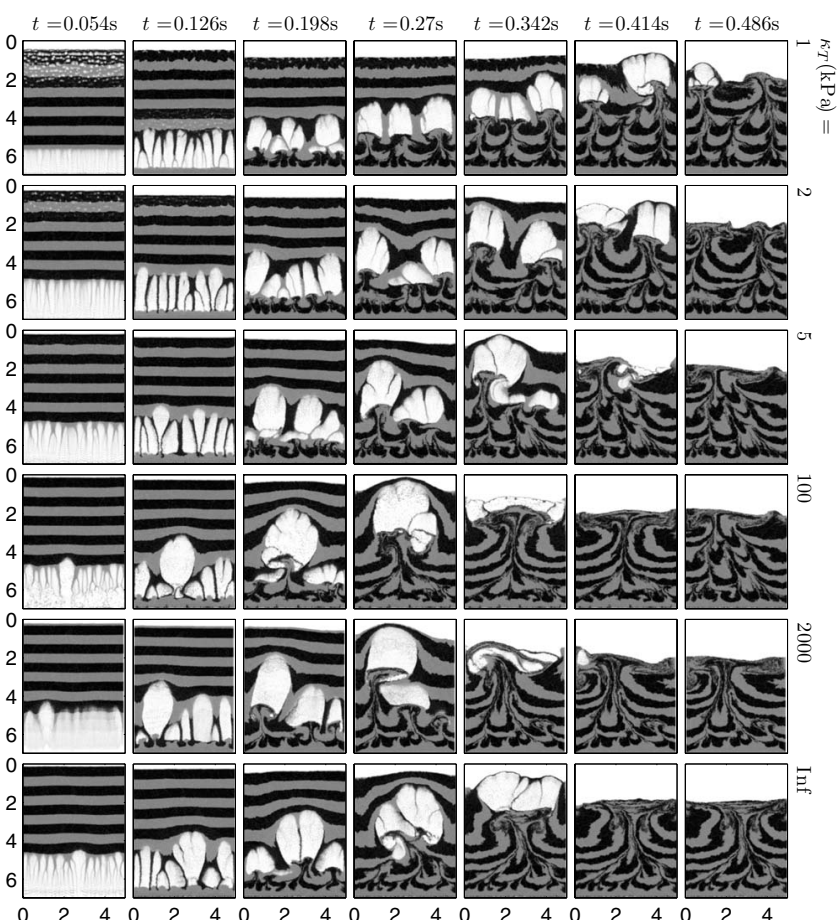

FIG. 2. Comparison of simulations with different fluid compressibilities at a fluid viscosity of air: $\mu_{f}($ air $)=0.0182 \mathrm{mPa}$. Gray and black areas represent areas filled with particles. The stripes are added artificially to better demonstrate the dynamics. From left to right time progresses in equal time steps and from top to bottom the bulk modulus is increased.

plotted the movement of the center of mass of all particles $\Delta R_{c}(t)=R_{c}(t)-R_{c}(0)$ in time. In the plots we observe oscillations of the top section for all the bulk moduli below $\kappa_{T}$ $\leq 5 \mathrm{kPa}$. Above this limiting bulk modulus, the movement of the center of mass does not seem to be influenced by the compressibility of the fluid. The oscillations are governed by the inertia of the mass of the grains in the top section and the elasticity of the fluid given by the bulk modulus. After the pressure rises in the bottom part of the cell fluid seeps through the porous media, exchanging momentum between the particles and the fluid and damping the oscillations. The system can show transient oscillations, which we observe for $\kappa_{T} \leq 5 \mathrm{kPa}$ or be in an overdamped regime for $\kappa_{T}>5 \mathrm{kPa}$.

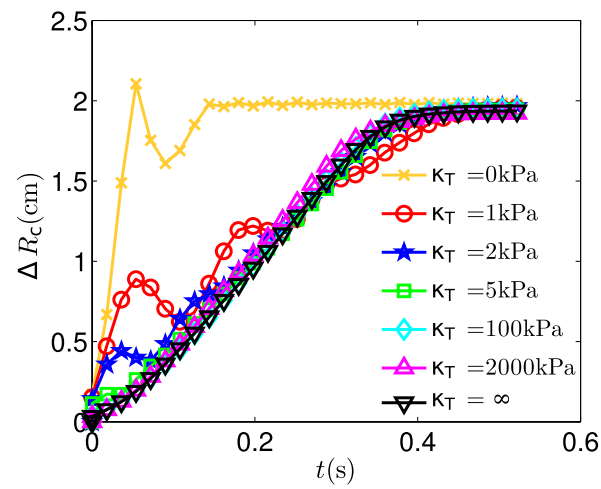

FIG. 3. (Color online) The $y$ position of the center of mass of all particles is plotted in time for different bulk moduli. 


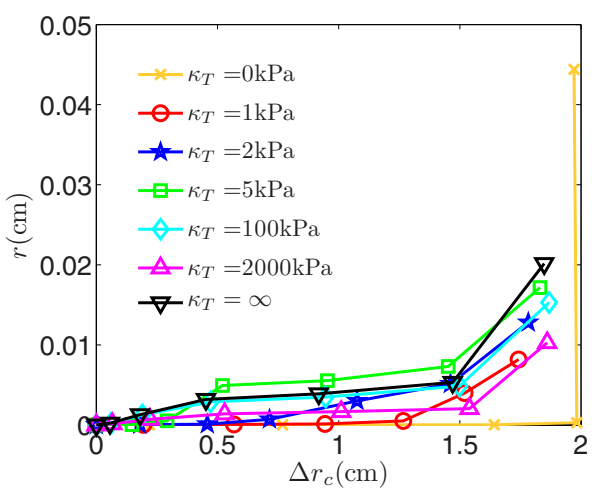

FIG. 4. (Color online) The excess path length $r(t)$ in Eq. (12) is plotted in time. Particles are falling mostly straight downward, and the bulk modulus hardly affects the excess path length.

In Fig. 4 we analyzed the excess path length $r(t)$ of the lowest layer of $N=8000$ particles at $t=0 \mathrm{~s}$, where the position of the $i$ th particle is given by $\mathbf{r}_{i}(t)$. The displacement of the center of mass of the lowest layer of $N=8000$ particles is $\Delta r_{c}(t)=r_{c}(t)-r_{c}(0)$, and we define the excess path length $r(t)$ as

$$
r\left(t_{s}\right)=\frac{\sum_{i}^{N} \sum_{j=1}^{s}\left|\mathbf{r}_{i}\left(t_{j}\right)-\mathbf{r}_{i}\left(t_{j-1}\right)\right|}{N}-\left[r_{c}\left(t_{s}\right)-r_{c}(0)\right],
$$

with the first sum over the particles and the second sum over the time steps $j$. If particles are falling straight with this definition in Eq. (12), the excess path length is zero. The excess path length is a measure of the complexity of the particle trajectories. The time resolution is small enough that no significant deviation was found when we only used every second time step instead of every time step.

In Fig. 4 we plotted the average excess particle trajectory $r(t)$ in relation to the movement in the $y$ direction of the center of mass $\Delta r_{c}$. We notice that the excess trajectory is almost zero until the particles hit the bottom of the cell at $r_{c}=1.6 \mathrm{~cm}$. This shows that the particles of this lower layer are falling mostly straight through the gap, independent of the compressibility of the fluid.

\section{B. When does compressibility become important?}

To estimate when compressibility becomes an important factor and affects the dynamics, we have to check two conditions: first if the weight of the packing of grains is enough to significantly compress the fluid in the base. In Eq. (5) this means that the pressure difference is comparable to the background pressure $P_{0}=\kappa_{T}$ for an ideal gas. In this case we experience oscillations of the top section if friction is neglected like in our case for a bulk modulus $\kappa_{T} \leq 5 \mathrm{kPa}$. Second we can define a skin depth for the pressure drop inside the porous matrix. For this simplified analysis we start with Eq. (6) and work in a reference frame moving with the particles. We assume small deformations of this falling particle plug, hereby neglecting the relative motion between the particles. We further take the solid fraction to be homogeneous

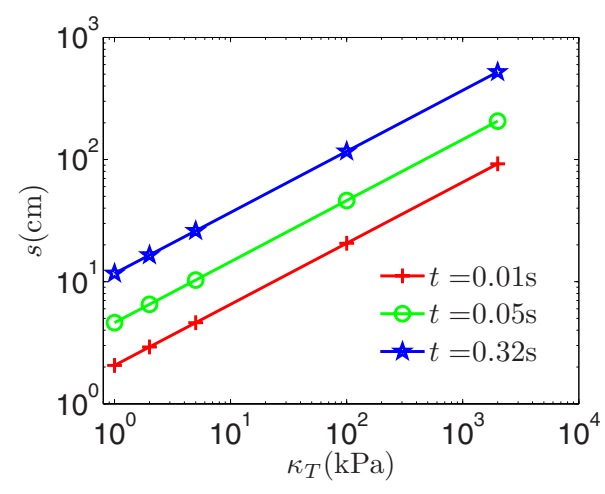

FIG. 5. (Color online) The skin depth for different bulk moduli at time steps connected with the oscillations: $t=0.01 \mathrm{~s}$ and $t$ $=0.05 \mathrm{~s}$. At the time that the top layer takes to fall through the fluid $t=0.32 \mathrm{~s}$, the skin depth is much larger than the system size.

$\nabla \frac{\kappa}{\mu}=0$ and that the pressure difference in the cell is small compared to the background pressure $P_{0}$. This gives $\hat{P} \approx \kappa_{T}$ and Eq. (6) simplifies to a diffusion equation:

$$
\frac{\partial P}{\partial t}=\frac{\kappa_{T} \kappa}{\phi \mu} \Delta P .
$$

The fundamental solution to this equation is

$$
P(x)=\frac{1}{\sqrt{4 \pi D t}} e^{-x^{2} / 4 D t},
$$

where $D=\kappa_{T} \kappa / \phi \mu$. In Eq. (14) we can define a skin depth $s$ where the pressure has decayed by $P(s)=\frac{1}{e}$. This skin depth is given by $s=\sqrt{4 D t}$. We can now compare this skin depth with the size of the Hele-Shaw cell. For typical values of $\rho_{s}=0.6, \mu_{f}($ air $)=0.018 \mathrm{mPa} \mathrm{s}$, and $t=0.01 \mathrm{~s}, t=0.05 \mathrm{~s}$, and $t=0.32 \mathrm{~s}$, we find a skin depth as shown in Fig. 5. The first two time steps correspond to the time steps that are in the range of the observed pressure oscillation period. The last is the time that the top layer takes theoretically to fall through the fluid gap, when the velocity of the top layer is given by the Darcy velocity $v_{d}=\left(\kappa / \mu_{f}\right) \nabla P$, and the pressure force balances the weight of the grains $\nabla P=\rho_{s} \rho_{m}$ g for a solid fraction of $\rho_{s}=0.6$. In this Fig. 5 we have calculated the skin depth $s$ for different bulk moduli. At the time that the top layer takes to fall through the fluid $t=0.32 \mathrm{~s}$ the skin depth is much larger than the system size for all $\kappa_{T}$. At the times connected with the oscillations the figure shows that the skin depth is in the range of the system size for all $\kappa_{T} \leq 5 \mathrm{kPa}$. For $\kappa_{T}>5 \mathrm{kPa}$ the skin depth gets much larger than the system size of $7 \mathrm{~cm}$. If we compare now the plot of the density field in Fig. 2, we can see that the lower compressibility affects rather the system when the skin depth is smaller than the size of the Hele-Shaw cell. When the packing of beads starts to fall downward, the pressure in the bottom of the cell will increase while in the top of the cell the pressure decreases. In the highly compressible case and for a homogeneous layer of beads the solution of the simple diffusion equation (14) has a curved profile and a skin depth smaller than the system size as shown in Fig. 6. Since the volume of fluid in the bottom of the cell is much larger than 


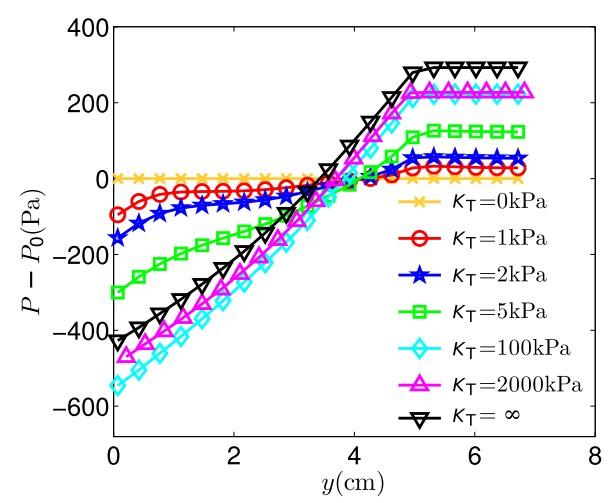

FIG. 6. (Color online) The averaged pressure profile as a function of the depth at $t=0.02 \mathrm{~s}$.

in the top, the movement of the particles will cause the pressure to drop faster in the top of the cell than the pressure increases in the bottom of the cell. This underpressure in the top part of the cell will strongly slow down the falling of the uppermost particles where the pressure gradient is the strongest. Inside the packing, away from the upper layer, the pressure gradient decreases and so does the upward force on the particles. Due to this decrease in the acceleration on the particles, the layer expands in the top part, creating the bubbles of low particle density. If on the contrary the skin depth is larger than the system size, the pressure profile becomes linear and the pressure gradient the acceleration of the beads is constant. The packing shows no noticeable expansion apart from its decompaction happening at its lower boundary, which is a different effect. The oscillations of the top layer can be described in a simplified way through a differential equation equivalent to a damped harmonic oscillator. The particles in the top layer shall be considered as a piston with a constant permeability and no relative movement of the particles. The initial empty volume in the bottom part is given by $V_{b}(0)$ and the top part by $V_{t}(0)$. The thickness in the $y$ direction of the top layer is $L$ and the cross-section area is $A=\Delta x h$. Defining $Y(t) \approx \Delta R_{c}(t)$ as the change in the position of the center of mass of all particles and $M$ as their total mass, we can state

$$
M \ddot{Y}-M g=-A\left(P_{b}-P_{t}\right) .
$$

In this simplified picture the cell consists of two compartments separated by a porous piston. The pressure gradient inside the piston is assumed to be constant and equal to the overall pressure gradient between the two compartments corresponding to the long-term limit of the pressure profile if the fluid is compressible. The change in the fluid volume in the bottom compartment $V_{b}(t)-V_{b}(0)$ is negative to the change in fluid volume in the top compartment $V_{b}(t)$ $-V_{b}(0)=-\left[V_{t}(t)-V_{t}(0)\right]$. There are two possible mechanisms affecting the fluid volumes. First is the compression or expansion of the fluid due to the movement of the piston, and second is the flow through the porous piston. This leads to the following expression for the fluid volume in the bottom compartment:

$$
V_{b}(t)-V_{b}(0)=\left[-A Y+\frac{A \kappa}{\mu} \int_{0}^{t} \frac{P_{b}-P_{t}}{L} d t\right] .
$$

With the definition of the bulk modulus $\kappa_{T}=-V \frac{\partial P}{\partial V}$, the pressure difference between top and bottom parts can be calculated as

$$
\begin{aligned}
P_{b}-P_{t}= & -\frac{\kappa_{T}}{V_{b}(0)}\left[-A Y+\frac{A \kappa}{\mu} \int_{0}^{t} \frac{P_{b}-P_{t}}{L} d t\right] \\
& -\frac{\kappa_{T}}{V_{t}(0)}\left[-A Y+\frac{A \kappa}{\mu} \int_{0}^{t} \frac{P_{b}-P_{t}}{L} d t\right] .
\end{aligned}
$$

Using now Eq. (15) in Eq. (17) and integration results in the differential equation of a damped harmonic oscillator:

$$
\ddot{Y}+\alpha \dot{Y}+\beta Y=\gamma t+g,
$$

where the constants are defined by

$$
\begin{aligned}
& \alpha=\frac{\kappa \kappa_{T} A}{\mu L}\left(\frac{1}{V_{b}(0)}+\frac{1}{V_{t}(0)}\right), \\
& \beta=\frac{\kappa_{T} A^{2}}{M}\left(\frac{1}{V_{b}(0)}+\frac{1}{V_{t}(0)}\right), \\
& \gamma=\frac{\kappa \kappa_{T} A g}{\mu L}\left(\frac{1}{V_{b}(0)}+\frac{1}{V_{t}(0)}\right) .
\end{aligned}
$$

With a standard ansatz $Y(t)=e^{\lambda t}$ in Eq. (18) two solutions of the homogeneous equation are found:

$$
\lambda_{1,2}=-\frac{\alpha}{2} \pm \sqrt{\frac{\alpha^{2}}{4}-\beta} .
$$

The system will be overdamped if the square root of Eq. (20) is positive, and oscillations only occur if the square root is negative. This is the case if

$$
\frac{\kappa^{2} \kappa_{T} M}{4 \mu^{2} L^{2}}\left(\frac{1}{V_{b}(0)}+\frac{1}{V_{t}(0)}\right)<1 .
$$

Assuming a system with the constants used in the simulations Eq. (21) predicts a critical bulk modulus of $\kappa_{T}$ $=589.3 \mathrm{kPa}$ for the transition from an overdamped to a damped system. Here, we furthermore assumed that $V_{b}(0)$ $=V_{t}(0)=A h(1.0 \mathrm{~cm})$ and a solid fraction of $\rho_{s}=0.6$. If the initial volume is $V_{t}(0)=A h(0.1 \mathrm{~cm})$ and $V_{b}(0)$ $=A h(1.9 \mathrm{~cm})$ the critical bulk modulus is $\kappa_{T}=111.9 \mathrm{kPa}$. Recalling Fig. 3 it can be seen that the transition occurs at comparable values in the simulations.

\section{Effect of the viscosity on the granular Rayleigh-Taylor instability}

In Fig. 7 the influence of the fluid viscosity $\mu_{f}$ is demonstrated in plots of the density field in black and white, where black stands for high particle density. Stripes in gray were added to emphasize the particle dynamics. The viscosity is changed from a value close to the viscosity of air $\mu_{f}$ (air) 


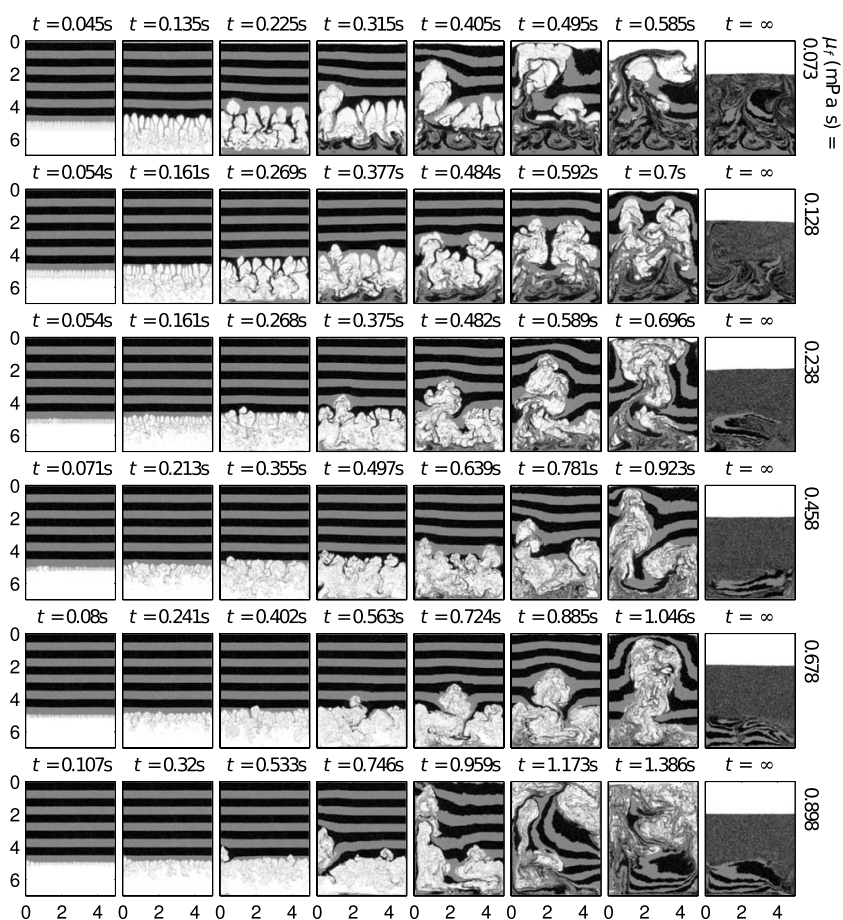

FIG. 7. The particle density field of simulations with different viscosities. From left to right time is progressing, and from top to bottom the viscosity is increased. If not specified the axis units are given in centimeters. White areas represent particle-free areas. Bubbles of low particle density can be observed.

$=0.018 \mathrm{mPa} \mathrm{s}$ and increased in steps with increasing step size. When the viscosity is increased a clear difference in the dynamics in the cell can be observed. The structures get smaller and the evolution of the dynamics is slowed down. When the viscosity is increased, the fingers finally disperse before they have reached the base of the cell. In the further progress the most advanced bubble of low particle density accelerates until the top section of compacted grains has been broken through. After this breakthrough the top section gets unstable, and the remaining blocks of compacted grains remain compacted while falling downward. This is a rather different dynamics, where we have already observed that the friction with the side plates is an important parameter [12].

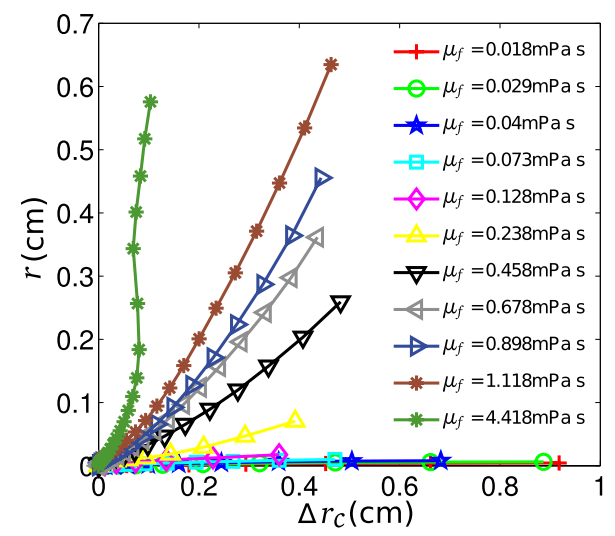

FIG. 8. (Color online) The average excess particle trajectory in relation to the displacement of the center of mass.

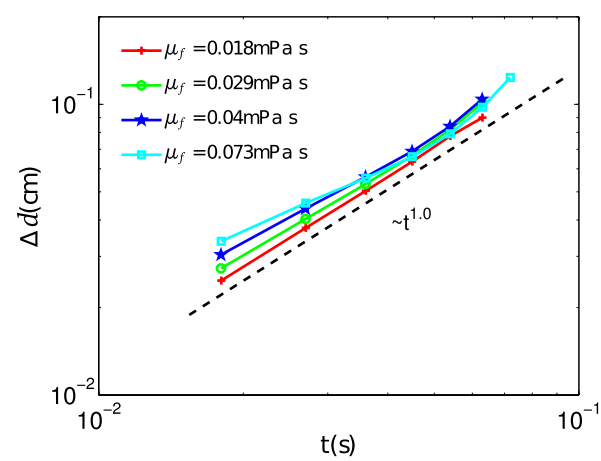

FIG. 9. (Color online) $\Delta d$ the average distance of particle pairs in time for low-viscosity fluids in bilogarithmic representation. The power-law fit with an exponent $b=1.0$ shows ballistic behavior.

The focus of this paper shall be kept on the start of the Rayleigh-Taylor instability, which is less sensitive to boundary conditions, and allows us to concentrate on the parametric study of the viscosity and compressibility effects. Thus, we have chosen not to study in detail this final stage of the dynamics, and we concentrate on the beginning of the simulations when the top layer is still intact.

The analysis of the excess path length of Sec. III A in Eq. (12) performed on the simulations with different viscosities leads to the plot in Fig. 8. Here, we can see that the first 8000 particles follow a longer trajectory in relation to the movement of their centers of mass the more viscous is the fluid. There is no simple way to rescale all the plots and collapse them for all viscosities. This shows that the characteristics of the patterns in the density field and the dynamics of the particles are changed due to the viscosity.

To further analyze this change in the mixing dynamics, we define $\Delta d$ as the average relative distance of particles pairs. These pairs were at time $t=0 \mathrm{~s}$ separated by a distance $\Delta d_{s}<0.028 \mathrm{~cm}$, which corresponds to two particle diameters. In this average only the first 600 particles are considered corresponding to the first two layers. The reason for this is that the front where particles disperse from the top section travels slower for higher viscosity, and the amount of par-

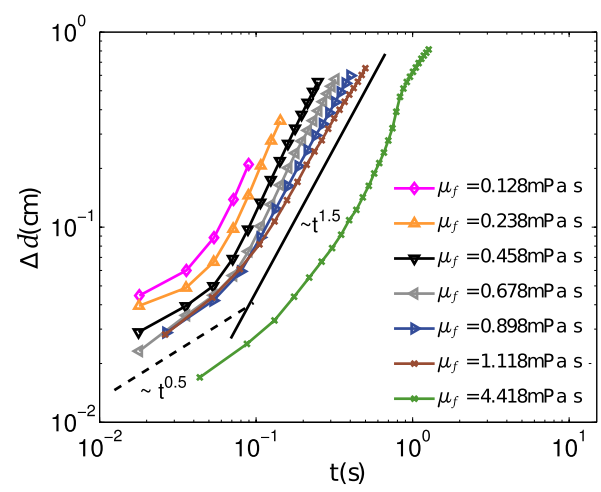

FIG. 10. (Color online) $\Delta d$ the average distance of particle pairs in time for high-viscosity fluids in bilogarithmic representation. The initial separation of the pairs has a diffusive behavior with an exponent close to $b=0.5$ in the dashed line. In the progress a crossover to a turbulent-dispersive behavior is observed with a slope close to $b=1.5$ in the solid line. 


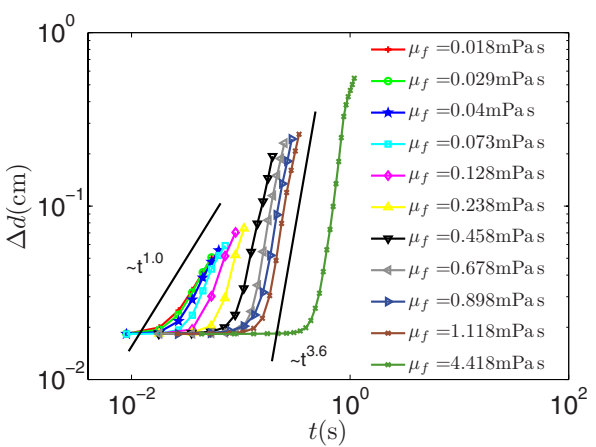

FIG. 11. (Color online) Bilogarithmic representation of $\Delta d$, the average distance of particle pairs in time, for a layer of 600 particles at a height of 2000 particles up in the packing. The slopes increase systematically with the fluid viscosity.

ticles that contribute to the averaged particle pair distance would depend on the speed the front travels with. Furthermore the dynamic patterns in the cell also depend on the height from the bottom of the cell and coarsen in time. By taking the average over the first 600 particles, it can be ensured that all the particles almost start moving instantaneously. The analysis stops when the first particle has reached a distance of $0.14 \mathrm{~cm}$ to the base of the cell corresponding to ten particle diameters. The average distance between the particle pairs grows in time while the particles are falling through the fluid as shown in the bilogarithmic representations in Figs. 9 and 10. The pair separation can be classified into two regimes. The first regime for lowly viscous fluids with $0.018 \leq \mu_{f} \leq 0.073 \mathrm{kPa}$ s shows a nonhydrodynamic or ballistic behavior where the exponent is close to $b=1.0$ in a power-law fit of $\Delta d=a t^{b}$. The particles in this regime fall with a constant relative velocity. In the second regime for highly viscous fluids with $0.128 \leq \mu_{f}$ $\leq 4.418 \mathrm{kPa}$ s the particle pairs follow an initial diffusive separation with an exponent close to $b=0.5$ before they enter the turbulent-dispersive behavior with an exponent close to $b=1.5$. Interestingly the latter exponent of $b=1.5$ corresponds to the Richardson law that predicts an exponent of $b=1.5$ for particle pair separation in fully developed turbulence [20-24]. For later stages this observation however changes. Looking at a layer of 600 particles at a height of 2000 particles up in the packing, the pair separation displays a slope of around $b=3.6$ for particles emerged in highviscosity fluids (see Fig. 11). The ballistic behavior for particles emerged in low-viscosity fluids is slightly more stable

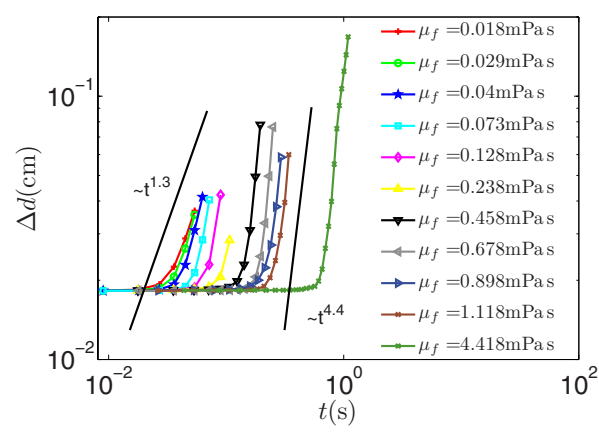

FIG. 12. (Color online) Bilogarithmic representation of $\Delta d$, the average distance of particle pairs in time, for a layer of 600 particles at a height of 4000 particles up in the packing.

and holds for a layer of 600 particles until a height of 4000 particles up in the packing (see Fig. 12). In both plots the slopes increase systematically with the fluid viscosity. In addition to the coarsening of the dynamic pattern the more rugged interface at later stages can cause this behavior. The more rugged interface at later stages causes the particles to disperse from the compacted layer at different times and can also contribute to a higher slope of the pair separation in the bilogarithmic representation.

\section{CONCLUSION}

As a conclusion we can state that the compressibility of the interstitial fluid affects the dynamical patterns much less than the viscosity. Under the conditions discussed in Sec. III B that the skin depth of the pressure is larger than the system size and that the weight of the grains does not lead to a significant compression of the fluid in the empty zone of the cell, the compressibility can be neglected. This results in an increase in the computational speed by a factor of around 20 for the present model from 480 to $24 \mathrm{~h}$ on a cluster with eight nodes. In the second part of this paper the viscosity was proven to have a strong effect on the dynamics of the particles. In terms of the mixing behavior the increase in the additional path length due to the increase in the fluid viscosity will result in a better mixing of the particles the more viscous the fluid is if internal friction is negligible. For some regions in the flow next to the initial fluid-grain pack boundary, a transient power-law behavior is observed for the dependence on time of particle pair separation. The exponent characterizing these power laws is observed to increase as a function of the fluid viscosity.
[1] P. Tegzes, T. Vicsek, and P. Schiffer, Phys. Rev. Lett. 89, 094301 (2002).

[2] R. M. Iverson, M. Logan, and R. P. Denlinger, J. Geophys. Res. 109, F01015 (2004).

[3] R. P. Denlinger and R. M. Iverson, J. Geophys. Res. 109, F01014 (2004).

[4] J. Duran and A. Reisinger, Sands, Powders, and Grains: An
Introduction to the Physics of Granular Materials (SpringerVerlag, New York, 1999).

[5] L. Bergougnoux, S. Ghicini, E. Guazzelli, and J. E. Hinch, Phys. Fluids 15, 1875 (2003).

[6] E. Guazzelli, Phys. Fluids 13, 1537 (2001).

[7] H. Nicolai, B. Herzhaft, E. J. Hinch, L. Oger, and E. Guazzelli, Phys. Fluids 7, 12 (1995). 
[8] H. Nicolai and E. Guazzelli, Phys. Fluids 7, 3 (1995).

[9] J. L. Vinningland, Ø. Johnsen, E. G. Flekkøy, R. Toussaint, and K. J. Måløy, Phys. Rev. Lett. 99, 048001 (2007).

[10] J. L. Vinningland, Ø. Johnsen, E. G. Flekkøy, R. Toussaint, and K. J. Måløy, Phys. Rev. E 76, 051306 (2007).

[11] J. L. Vinningland, Ø. Johnsen, E. G. Flekkøy, R. Toussaint, and K. J. Måløy, Phys. Rev. E 81, 041308 (2010).

[12] M. J. Niebling, E. G. Flekkøy, K. J. Måløy, and R. Toussaint, Phys. Rev. E 82, 011301 (2010).

[13] Ø. Johnsen, R. Toussaint, K. J. Måløy, and E. G. Flekkøy, Phys. Rev. E 74, 011301 (2006).

[14] Ø. Johnsen, C. Chevalier, A. Lindner, R. Toussaint, E. Clément, K. J. Måløy, E. G. Flekkøy, and J. Schmittbuhl, Phys. Rev. E 78, 051302 (2008).

[15] S. McNamara, E. G. Flekkøy, and K. J. Måløy, Phys. Rev. E

$$
\text { 61, } 4054 \text { (2000). }
$$

[16] D.-V. Anghel, M. Strauss, S. McNamara, E. G. Flekkøy, and K. J. Måløy, Phys. Rev. E 74, 029906(E) (2006).

[17] P. C. Carman, Chem. Eng. Res. Des. 75, S32 (1997).

[18] E. G. Flekkøy, R. Delgado-Buscalioni, and P. V. Coveney, Phys. Rev. E 72, 026703 (2005).

[19] W. H. Press and W. T. Vetterling, Numerical Recipes (Cambridge University Press, Cambridge, England, 2002).

[20] L. F. Richardson, Proc. R. Soc. London, Ser. A 110, 709 (1926).

[21] T. H. Dupree, Phys. Fluids 9, 1773 (1966).

[22] T. H. Dupree, Phys. Fluids 15, 334 (1972).

[23] G. Boffetta, A. Celani, A. Crisanti, and A. Vulpiani, EPL 46 , 177 (1999).

[24] J. H. Misguich and R. Balescu, Plasma Phys. 24, 289 (1982). 